\title{
TU4E-(SS1)-5
}

\section{EFFECTS ON EMISSION PROPERTIES OF INTERFACE THIN LAYERS IN InGaN/GaN QUANTUM WELL STRUCTURES}

\author{
Yung-Chen Cheng, Horng-Shyang Chen, $\underline{C}$. C. Yang, and Zhe-Chuang Feng \\ Graduate Institute of Electro-Optical Engineering and Department of Electrical Engineering, \\ National Taiwan University, 1, Roosevelt Road, Section 4, Taipei, Taiwan \\ (phone) 886-2-23657624 \\ (fax) 886-2-23652637 \\ (e-mail)ccy@cc.ee.ntu.edu.tw \\ Gang Alan Li \\ LED Expert Corporation, Kaohsiung, Taiwan
}

Abstract - We report the variations of optical characteristics in InGaN/GaN quantum well samples with different thin layer (about $1 \mathrm{~nm}$ ) structures between the wells and barriers, including silicon-doped InN and InGaN compounds.

In this paper, we compare the photoluminescence (PL) and electro-luminescence (EL) properties of four InGaN/GaN QW samples with different well/barrier interface structures. Thin layers of about $1 \mathrm{~nm}$ in thickness and different compositions were inserted between a well and the neighboring barriers. It is found that with either InGaN or InN thin layers, silicon-doped or un-doped, the photon emission efficiency is improved, when compared with a standard InGaN/GaN QW sample with barrier doping. The samples were grown on c-plane sapphire with MOCVD. After a 4- $\mu \mathrm{m}$ Si-doped GaN $\left(4 \times 10^{18} \mathrm{~cm}^{-3}\right.$ in doping concentration) layer, eight periods of QW were grown with well width $2.5 \mathrm{~nm}$ and barrier width $13.5 \mathrm{~nm}$. The barrier is Si-doped with $1 \sim 5 \times 10^{17}$ $\mathrm{cm}^{-3}$ in doping concentration. The nominal indium content of the InGaN wells was estimated to be $20 \%$. On top of the QW layers, a $150 \mathrm{~nm}$ p-GaN layer $\left(3 \times 10^{17} \mathrm{~cm}^{-3}\right.$ in carrier concentration) was grown for Ohmic contact. The sample with aforementioned structure is designated as sample 344 . In sample 341 , a Si-doped InN thin layer of about $1 \mathrm{~nm}$ in thickness is inserted between a well and its neighboring barrier on either side. In sample 343, instead of doped InN, doped InGaN thin layers were used. The nominal indium content is the same as that in wells. Then, in sample 345 , an un-doped InN thin layer of about $1 \mathrm{~nm}$ in thickness is inserted between wells and barriers. Figs. 1-4 show the EL/PL spectral peak positions as functions of temperature of samples $341,343,344$, and 345 , respectively. It is noted that although we briefly called them peak positions, part of data points were obtained through the calculations of the spectral center of mass because the Fabry-Perot oscillations make the identification of the spectral peaks difficult. It is interesting to see that in all the four samples, EL and PL peaks are different in the concerned temperature range. In EL measurements, the possibly minimum injection currents were used in various samples. The injection currents for samples $341,343,344$, and 345 are $0.7,0.5,1.5$, and $0.74 \mathrm{~mA}$, respectively. Among Figs. 1-4, one can see that all the four samples show dramatic EL peak variations with temperature. Except sample 343, the other three samples show tremendous red shifts when temperature rises beyond $150 \mathrm{~K}$. In samples 341 and 345 , EL spectra show blue shifts when compared with PL spectra. However, in sample 343 , red shifts are observed. Then in sample 344, PL peaks are located between the high and low energy sides of EL peak. Regarding PL spectral peaks, all the four samples show, more or less, S-shape temperature variations. The flat PL curve in Fig. 1 below $150 \mathrm{~K}$ also indicates the blue shift trend and hence the tendency of forming the $\mathrm{S}$ shape variation in sample 341. It is interesting to note that in all the four samples, steep EL peak variations always occur in the temperature ranges of significant blue shifts in PL spectra. The only difference is that in sample 343 , EL peak blue shifts and in the other three samples, EL peak red shifts. Actually, the trend of the EL curve in Fig. 2 follows well that of the PL curve. In samples 344 and 345, the trends of EL and PL are basically reversed.

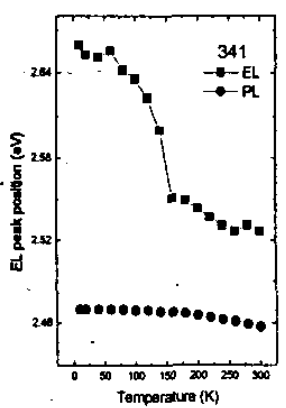

Fig. 1

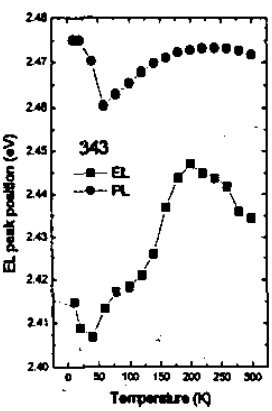

Fig. 2

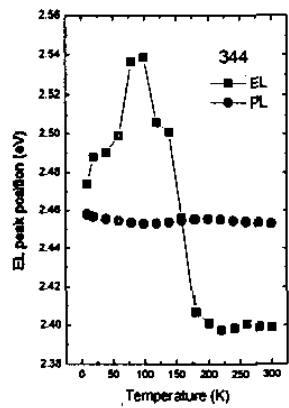

Fig. 3

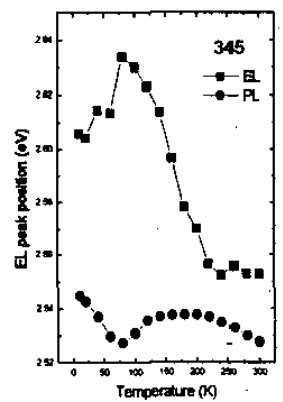

Fig. 4 